\title{
La diction du tragédien ridicule sous l'Ancien Régime
}

Sabine Chaouche

\section{(2) OpenEdition}

1 Journals

Édition électronique

URL : http://journals.openedition.org/studifrancesi/6166

DOI : 10.4000/studifrancesi.6166

ISSN : 2421-5856

Éditeur

Rosenberg \& Sellier

\section{Édition imprimée}

Date de publication : 1 novembre 2010

Pagination : 497-507

ISSN : 0039-2944

\section{Référence électronique}

Sabine Chaouche, "La diction du tragédien ridicule sous I'Ancien Régime », Studi Francesi [En ligne], 162 (LIV | III) | 2010, mis en ligne le 30 novembre 2015, consulté le 07 janvier 2021. URL : http:// journals.openedition.org/studifrancesi/6166 ; DOI : https://doi.org/ERREUR PDO dans /localdata/ www-bin/Core/Core/Db/Db.class.php L.34 : SQLSTATE[HY000] [2006] MySQL server has gone away

\section{(c) (i) $\odot$}

Studi Francesi è distribuita con Licenza Creative Commons Attribuzione - Non commerciale - Non opere derivate 4.0 Internazionale. 


\section{La diction du tragédien ridicule sous l'Ancien Régime}

L'un des «procédés» comiques récurrents dans les pièces des forains et plus tard, de l'Opéra-Comique, est la parodie de la déclamation tragique. Une image s'est peu à peu figée dans le théâtre du XVIII siècle, à la fois intense et ambiguë: intense parce qu'elle dénonçait avec une certaine violence, les défauts des tragédiens de la Comédie-Française en les transformant en de simples bouffons (les tragédiens étaient ainsi rabaissés et devenaient semblables à ceux qu'ils honnissaient le plus: les histrions du Pont-Neuf ou les «bateleurs» des troupes de la foire ou de province ${ }^{1}$ ); ambiguë en ce qu'elle était le produit de l'imagination, et de différents auteurs, et des acteurs interprétant la caricature.

Quel sens donner dès lors à la parodie de la diction tragique et à la caricature du tragédien? Peut-on dire qu'elles reflètent, de manière relativement fidèle, les pratiques du temps, et qu'elles seraient ainsi moins déformations comiques que «portraits ressemblants»? Comment comprendre la persistance d'un «anti-modèle» tragique, de la critique d'une diction «non naturelle» depuis L'Impromptu de Versailles de Molière?

Les critiques adressées aux tragédiens n’ont été jusqu'ici réellement confrontées aux théories sur l'art de l'acteur, ni même confrontées au contexte historique. Ainsi par exemple on ne peut nier que l'article de Nathalie Rizzoni, Du «je» au jeu de l'acteur au XVIII siècle ou l'art du comédien par lui-même est extrêmement riche en références. Il éclaire de manière précise les pièces où apparaissent les caricatures. Mais les travers des tragédiens ne sont absolument pas mis en perspective avec l'évolution du jeu de l'acteur, ni des théories, ni même avec les realia scenica. L'article se contente d'être descriptif, s'attachant essentiellement à renforcer les «topoï» sur le tragédien, sans discuter de la véracité ou de la vraisemblance, ou non, des portraits créés par les forains. Certes, cette démarche a certaines vertus: celle de réhabiliter le théâtre de la foire, d'en montrer toute la singularité et la richesse; celle de rendre aux acteurs le statut de professionnels des arts du spectacle. Loin de vouloir dénier ces aspects du théâtre de la foire et de ses acteurs, nous désirons, ici, nuancer cette vision trop tranchée, et interroger aux mieux les textes afin d'explorer de nouvelles pistes de lectures ${ }^{3}$.

(1) S’il nous est resté l'image du pauvre hère des troupes de campagne à travers le roman comique de Scarron, d'autres portraits des troupes de campagne nous sont parvenus à travers les Mémoires de Jean Monnet, en particulier.

(2) «L'Annuaire théâtral», 42, 2007, pp. 91-105.

(3) Des travaux importants sur la foire ont été menés ces dernières années. Barry Russell et David Trott furent parmi les premiers à s'être intéressés à ce type de théâtre. Des études capitales ont été publiées ces dernières années comme par exemple: David TROTT, Théatre du XVIII siècle: jeux, écritures, regards. Essai sur les spectacles en France de 1700 à 1790, Montpellier, Espaces 34, 2002; Isabelle Martin, Le Théâtre de la Foire, Oxford, SVEC, 2004; Nathalie Rizzoni, Charles-François Pannard et l'esthétique du 'petit', Oxford, SVEC, 2000. Voir aussi les anthologies de Françoise RubELlin et les travaux de son centre de recherche à Nantes (www. foires.net). 
Dans leurs pièces, les forains stigmatisent la diction des tragédiens qu'ils jugent trop cadencée à leur goût, et qui semble privilégier la forme au détriment du sens des vers ${ }^{4}$. Sur les scènes de la foire, elle se veut un effet comique et un jeu de scène. En effet, les tragédiens de la Comédie-Française apparaissent outranciers, monotones et artificiels. C'est ce que suggèrent les différents noms dont les personnages sont affublés: Monsieur l'Amphigouri dans L'Assemblée des acteurs de Charles François Pannard et Carolet (1737), ou Métromane dans Acajou (1744) ou Ampulas dans La Ressource des Théâtres (1760), opéra-comiques de Charles-Simon Favart. Comme l'indique Nathalie Rizzoni les pièces où la déclamation emphatique est tournée en ridicule sont nombreuses. Elle cite entre autres, L'Ombre de la Foire (1720), Les Désespérés $(1732-1737)^{5}$.

La monotonie de la diction se traduit par un jeu sur les mots et la forme poétique. En effet, certains hémistiches sont systématiquement remplacés par un groupement de syllabes jouant sur les allitérations (voyelle orale «a» alternant avec la voyelle nasale «an»; succession de sons qui semblent évoquer le braiment d'un âne) et les consonances rudes (ainsi des consonnes avec l'occlusive dentale «t» et la constrictive «r»-dorso-vélaire probablement - qui donnent à la diction un air militaire ou tout du moins, musicalement l'air d'une «marche»): «Tarantan tan Tarantan tan taran tan tan», «Ton teron ton teron ton!», «Taratanta, vertu, taratantara, crime. / Taratantara, poignard, taratanta, victime» ${ }^{6}$. Il semble ainsi que l'image du tragédien ressortisse à une forme de grotesque-comique.

Or ces substitutions phonétiques habiles, qui sont en soi assez similaires à des onomatopées ou à du charabia (un galimatias de sons), posent de réels problèmes d'analyse critique. En effet, la déclamation tragique, telle que nous la présentent les forains, est-elle une forme «grotesque» tant sur la scène de la Comédie-Française que sur la scène de la foire? Ou ne l'est-elle qu'à la foire uniquement? Quel est le degré de déformation subie? Ainsi la diction tragique, créée à la foire, devient-elle «monstrueuse» et donc risible? La diction raillée est-elle alors étrangère à la «réalité» historique? Ou devient-elle «étrange» sur toutes les scènes, parce que les codes et conventions ne sont plus compris ou prisés? En ce sens, comment rendre compte avec justesse de la manière dont elle est interprétée, jouée, et son effet sur le public tant à la foire qu'à la Comédie-Française? La déclamation paraît-elle inquiétante, incompréhensible, absurde? Est-elle finalement une hypertrophie créatrice ou simple déformation des pratiques du temps? Y a-t-il même automatisation de la caricature dans la mesure où une invariabilité apparaît d'une pièce foraine à l'autre (la reprise plus ou moins fidèle des mêmes syllabes: «taratata», «taratantata»). Ainsi on passe progressivement du foisonnement imaginatif au manque d'imagination (on recycle en effet le cliché de la diction tragique élaboré dans L'Impromptu de Versailles, on l'accommode et l'assaisonne à chaque nouvelle pièce). L'image devient-elle alors, à la longue, hybride, voire totalement imaginaire?

Ces interrogations appellent plusieurs remarques. D'une part, la forme de la caricature est à rapprocher d'une anecdote dramatique rapportée par Jean-Nicolas Servandoni D'Hannetaire, directeur du Théâtre de la Monnaie à Bruxelles, dans son ouvrage Observations sur l'art du comédien (paru tout d'abord en 1764, puis réédité en 1774): «C'était surtout le vice de l'ancienne Déclamation: \& j’ai connu un vieux Comédien si habitué à faire sonner la rime et à cadencer les vers, qu'une fois dans ce

(4) L'idée de «perroquet» est émise par JeanFrançois Regnard et Charles Dufresny dans Les Chinois mais aussi par Molière dans L'Impromptu de Versailles lorsqu'il suggère que la déclamation de
Montfleury a trop de feu et ne s'accorde pas avec la situation représentée.

(5) N. Rizzoni, art. cité, p. 95.

(6) N. Rizzoni, art. cité, p. 95. 
passage de Mithridate: Quand le sort ennemi m'aurait jeté plus bas, / Vaincu, persécuté... ne se rappelant pas assez tôt le dernier hémistiche du second vers, ne put s'empêcher, par une certaine routine de la mesure, d'y substituer machinalement, Tati, tatou, tatas, sans discontinuer le reste de la Tirade et sans même se déconcerter...» ${ }^{7}$. D'après cette anecdote, ce type de déclamation cadencée accentuant fortement la rime est dépassé. À l'époque où est rédigé l'ouvrage, de nouveaux modes de diction sont apparus, plus conformes avec le besoin de réalisme scénique propre au XVIII siècle (les usages pourraient aussi différer d'un pays à l'autre: France/Pays-Bas autrichiens mais il semble que le renouveau de la diction ait été commun aux deux pays) ${ }^{8}$. Servandoni D'Hannetaire évoque donc un usage ancien, fait d'une autre génération d'acteurs, qu'il considère comme désuet. On peut supposer, Servandoni D'Hannetaire étant né en 1718, que l'événement a eu lieu bien après 1720 , c'est-à-dire après que sont apparues les premières caricatures jouant sur ces sonorités'. Faut-il croire alors que le comédien se soit inspiré lui-même des pièces foraines? On pourrait le penser si celuici jouait à Paris (D’Hannetaire ne le dit). La foire aurait ainsi créé de toute pièce cet «effet» comique (un «lazzi» en quelque sorte) et ce jeu de scène qui serait devenu par la suite, par le succès rencontré, une «recette» destinée à attirer des spectateurs et à faire du profit (un «filon» exploité en somme à des fins commerciales et financières). En outre, le récit suggère que le fait est ponctuel. «Tati tatou tata» rappelle certes les caricatures de la foire, mais ici la substitution se produit lorsque l'acteur a soudainement un trou de mémoire. Il représente un cas extrême de mauvais tragédien, ou tout du moins une désinvolture de l'acteur-ivrogne.

D'autre part il faut insister sur le fait que ces caricatures du tragédien semblent comme «figées». Elles donnent une vision de la déclamation tragique uniforme, et surtout, immuable. Or si l'on s'en tient exclusivement à cette «lecture» de la diction tragique, c'est tout une partie de l'histoire de la déclamation et du style des acteurs qui est occultée. En effet, c'est précisément entre 1720 et 1750 que se renouvellent rapidement les genres de diction. Certes les années 1720 succèdent à l'époque de la déclamation chantante incarnée par une Mlle Duclos dont la diction est devenue glapissante $^{10}$. Mais la décennie qui suit voit le retour de Baron et l'arrivée de Mlle Lecouvreur, qui, d'après l'ensemble des témoignages sur l'art théâtral, ont proprement révolutionné la manière de dire les vers. Baron est célèbre pour avoir suivi les préceptes de Molière, pour avoir su redonner du «sens» aux vers et pour avoir introduit des pauses dans ceux-ci, bref pour avoir su «parler en récitant» selon le mot de Marmontel $^{11}$. Il en va de même de la déclamation de Mlle Lecouvreur, jugée simple

(7) J.-N. Servandoni D'Hannetaire, Observations sur l'art du comédien, Paris, s.n., 1774, note de la page p. 208. La fin de l'anecdote est la suivante: «C'est cet Acteur qui, toute sa vie, accoutumé d'aller à la Buvette à chaque entracte d'une pièce, se trouva un jour si ivre à la fin de la même Tragédie, qu'en prononçant le dernier vers, venez, et recevez l'âme de Mithridate, il finit par offrir une image très dégoûtante de son personnage expirant surtout pour Xipharès, obligé d'en recevoir les derniers embrassements dans la pièce».

(8) Voir S. Chaouche, La Philosophie de l'Acteur, 1738-1801, Paris, Champion, 2007, pp. 174-213.

(9) Rappelons par exemple que L'Ombre de la Foire date de 1720.

(10) Voir S. Chaouche, La Poésie racinienne: chant ou déclamation?, Racine Poète, «La Licorne», 50, 1999, pp. 235-256; La diction poétique et ses enjeux sur la scène française, le passage de l'âge classique au siècle des Lumières, «Papers on French Seventeenth Century Literature», 31 (60), 2004, pp. 69-100.

(11) «Ce fut Baron l'élève de Molière, et l'instituteur de la belle déclamation. C'est son exemple qui va fonder nos principes; et nous n'avons qu'une réponse à faire aux partisans de la déclamation chantante: Baron parlait en déclamant, ou plutôt en récitant, pour parler le langage de Baron lui-même; car il était blessé du seul mot de déclamation. [...] La déclamation de Baron causa une surprise mêlée de ravissement» in S. CHAOUCHE, La diction théâtrale au XVIII siècle: 'déclamer' ou 'parler en récitant'?, «L'Information Littéraire», 3, 2000, p. 82. Noter, que, si le témoignage de Marmontel est tardif (dans les années 1750), il est en accord avec les témoignages du temps rapportant la manière dont Baron déclamait sur scène (témoignages, qui par leur consensus, ne semblent pas relever purement 
et non enflée. C'est ensuite au tour de Mlle Dumesnil d'innover sur la scène tragique dans les années 1740 par son jeu parfois familier, mais surtout impulsif ${ }^{12}$, sa diction volubile et véloce dans certains passages pathétiques ou intenses, émotionnellement, ayant frappé ses contemporains. Si Lekain débute par une déclamation tonitruante aux tons extraordinaires, il adoptera un style autre par la suite. Les acteurs vedettes de la Comédie-Française de la période concernée sont absents de la scène de la foire dans la mesure où la caricature ne vise pas, apparemment, un tragédien en particulier, mais un mode de diction jugé inapproprié au goût du temps.

Les forains ont-ils systématiquement tourné en ridicule les tragédiens de peur que leur public n'aille à la Comédie-Française admirer les nouveaux tragédiens et leurs styles modernes de diction? Ont-ils donc tenté de parer toute perte financière, leur position en tant que théâtre privé, étant, bien évidemment plus fragile que les théâtres officiels «subventionnés» et soutenus par la monarchie? Les pièces mettant en scène les tragédiens servaient-elles aussi de garde-fou aux acteurs du Français? Ainsi chaque mauvais tragédien aurait pu susciter une satire - d'où cet écart entre les dates de création des pièces citées précédemment $\left(1720,1732,1744^{13}\right)$.

Il est vrai que les quelques tragédiens «avant-gardistes» de la Comédie-Française ne reflètent qu'imparfaitement l'ensemble des pratiques scéniques du temps. Dans quelle mesure les conventions héritées de l'actio oratoire survivent-elles et sont-elles toujours en usage au XVIII ${ }^{\mathrm{e}}$ siècle? Les débats sur la diction qui se poursuivent tout au long du siècle (le terme «déclamation» symbolisant au fur et à mesure que l'on avance dans le siècle, une mauvaise manière de prononcer les vers) traduisent des préoccupations réelles, tant chez les penseurs que les acteurs, pour améliorer celle-ci (briser notamment son «refrain chantant»). Acajou, pièce la plus tardive, dénonce encore le «mécanisme» de la diction, où «malgré le sens commun» les acteurs sont «guidés par la mesure» et dont les tons sont «notés». Ils disent ainsi des vers «qui tombent deux à deux», parfois même les «mugissent» ou les «crient» (I.4). Il ne s'agit donc que «de faire du bruit» et des éclats pour impressionner le public.

Ces critiques ne diffèrent que peu de celles de certains rhétoriciens du siècle précédent, ou même du début du XVIII ${ }^{\mathrm{e}}$ siècle, à l'encontre des déclamateurs ${ }^{14}$. Ainsi Albert de Paris mentionne encore en 1701 cette manière de déclamer particulière fondée sur le retour cyclique de mêmes sonorités qui ôte toute vie à la déclamation. Elle est à rapprocher des deux «accents» parodiés par les forains: «Il est ici question de parler, \& non de chanter, \& je m'étonne de ce que des hommes faits n'ont pas la hardiesse de quitter une certaine cadence que les enfants suivent, lorsqu'ils veulent contrefaire des prédicateurs, en mesurant seulement certains nombres d'accents, sans prononcer de paroles. Il semble que ce soit là une flexibilité de voix mais ce n'est rien moins; car ces sortes de chanteurs n'ont que deux tons, desquels ils ne sortent jamais, sur lesquels ils retombent toujours alternativement. Mais ce qui est entièrement opposé à la flexibilité de la voix, c'est ce qu'on appelle Monotonie, lorsqu'on parle d'une manière unie, comme si on lisait» ${ }^{15}$. Les forains voulaient-ils débattre en réalité

de la légende ou du petit fait vrai enjolivé sous la plume d'un anecdotier). Voir La Scène en contrechamp, anecdotes françaises et traditions de jeu au siècle des Lumières, éd. S. Chaouche, Paris, Champion, 2005.

(12) S. Chaouche, La Philosophie de l'Acteur, édition citée, p. 203.

(13) Les Débuts de Dominique et Romagnesi (Théâtre Italien), de même que La Muse pantomime mettent aussi en scène les défauts des tragédiens.
(14) GUÉRET, Entretiens sur l'éloquence de la Chaire et du Barreau, Paris, J. Guignard, 1666, pp. 10-11 («Elle [la véritable Eloquence] est encore ennemie de l'Eloquence des Déclamateurs qui ne s'exerce que sur des faits inventés à plaisir, qui est toute bigarrée de figures, toute pleine d'emportements ridicules, \& qui n'a point d'autre règle que les caprices d'une imagination déréglée») et pp. 47-48.

(15) La Véritable manière de prêcher selon l'esprit, de l'Evangile, Paris, Nicolas Couterot, 1701, p. 319. 
$\mathrm{du}$ «chant» (le «vrai» chant versus le «faux» chant ou déclamation chantante ${ }^{16}$ ), ou des chants les plus aptes à plaire à un public populaire (c'est-à-dire la chanson guillerette des chansonniers versus le récitatif de l'Opéra)?

Il faut immédiatement ajouter ici que ces derniers, malgré le lien «naturel» unissant l'Opéra-comique à l'Opéra (le chant dans les pièces et le privilège de pouvoir chanter sur scène), n'hésitent pas à se moquer de l'Académie Royale de Musique. Souvent protectrice dans les pièces cependant qu'intéressée, elle apparaît néanmoins la risée des acteurs lorsqu'il est question de savoir plaire au public. Les noms des personnages symbolisant l'Opéra sont tout aussi évocateurs que ceux des tragédiens comme par exemple Narcotique (La Ressource des Théâtres), chantant son ennui d'être ennuyeux: «La tristesse m'accable: / Moi dont la gloire alloit aux cieux / Hélas! depuis long-temps on me trouve ennuyeux. / J'ai donné du galant, du badin, du terrible, / Mes cris ont fatigués la terre \& les enfers: / Vainement je fais l'impossible, / Mes airs sont à présent aussi froids que mes vers» (sc. 5). Dans Le Départ de l'OpéraComique le spectateur assiste à une satire de la tragédie lyrique, par Rebours et Hamoche: «J'ay vu l'amant d'une Bergere / Lorsqu'elle dormoit dans un bois / Prescrire aux oiseaux de se taire / Et luy chanter a pleine voix»; «J'ay vu par un destin bizarre / Les Heros de ce pays la / Se desesperer en becare / Et rendre l'ame en a mi la» ${ }^{17}$. Les fautes de vraisemblance et les trucages percent derrière le chant ou le jeu des chanteurs, et l'illusion n'est dès lors que relative.

La Nouvelle troupe de Charles-Simon Favart et l'abbé Claude-Henri Fusée de Voisenon ${ }^{18}$, présente une admirable scène mêlant habilement «déclamation pompeuse» (alexandrins, registre de langue soutenu et emphase) et guinguette, chanson populaire (vers hétérométriques, langage courant). Les didascalies indiquent d'ailleurs cet entrelacement entre le déclamé et le chanté (fait du même personnage ce qui prouve ainsi la virtuosité de Madame Favart tenant le rôle de Justine). A la scène 13, c'est un paysan qui fait part de son expérience à l'Opéra: «Et je m'souviens ben d'tout: j'crais entendre les vouas / Corner encore à mes oreilles. / Un biau Prince d'abord venoit chanter du nez; / Sa Princesse d'un air honnête, / Les bras en l'air, les yeux tornés, / Ly répondoit en chantant de la tête, / Et pis y avoit un Roua, droit comme un Estafier. / Stila chantoit d'la gorge, \& pis y avoit un chantre / Qui, pour se donner l'air sorcier, / S'battoit les flancs, tiroit la voix d'son ventre, / Ah! qu'c'étoit biau! Morgué, dis-je à part moua, / [...] S'il faut crier, j'crierons tout comme un autre, / Au plus fiar. [....] Oui, quand on gueul' ben fort, on dit: c'est excellent». Suit alors une discussion sur la prononciation. L'accent campagnard est évidemment malséant dans le genre lyrique, mais le paysan répond: «Mon accent est stila/ Don ton se sert à l'Opira; / Eh! pargué, c'est ce qu'on enseigne / Vous dirais: régne, Amour; fi donc; moi, / j'dirois: rêgne. / Rêgne, Amour. V'là qu'est pû ronflant. / Vive les sons qui remplissent la bouche. / C'n'est pas l'cœur qu'il faut que l'on touche, / C'est l'oreille, morguais». Puis il chante tantôt en haute-contre, tantôt en basse-taille. La leçon de diction «régne» > «rêgne» semble indiquer une ouverture plus grande de la bouche (passage du é au ê). Les tragédiens et les chanteurs lyriques accentuaient-ils l'aperture des sons vocaliques donnant ainsi plus d'emphase aux mots et, par la même occasion une intensité plus grande - mais qui semble, en contrepartie, contraire au parler naturel car guindée et affectée?

Ainsi les chanteurs apparaissent tout aussi artificiels et soporifiques que les tragédiens (image du chanteur qui émerge dès le XVII siècle avec Saint-Evremond), de

(16) Voir S. Chaouche, La Poésie racinienne: chant ou déclamation?, cit., pp. 235-256.

(17) BnF, Fonds des manuscrits français, 9323
[3417], sc. 7.

(18) La Nouvelle Troupe, Paris, s.d., sc. 11. 
même qu'ils semblent ne savoir aussi que hurler et crier sur scène. Ces moqueries valables tout autant pour le lyrique que le tragique (voire le tragico-lyrique) se veulent cependant «traditionnelles». Elles rappellent les parodies élaborées par Molière (les Grands Comédiens, par un jeu outré et cabotin souhaitant uniquement provoquer le brouhaha dans la salle) ou par La Bruyère ${ }^{19}$. Toutefois, elles sont aussi en accord avec les critiques des penseurs du temps de Pierre Rémond de Sainte-Albine (Le Comédien, 1747) à Claude-Joseph Dorat (La Déclamation théâtrale, 1766). L'acteur qui court après la gloire et les applaudissements est à peu près partout condamné pour ses excès et son charlatanisme. Doit-on pour autant considérer que tous les acteurs de la Comédie-Française sont des charlatans? Non, bien évidemment. Les reproches s'adressent plutôt aux amateurs ou aux tragédiens avides de gloire et de notoriété, ou ceux dont l'orgueil les pousse à croire qu'ils ont du génie. Tout comme au début du XVII ${ }^{\mathrm{e}}$ siècle Mazarin se moquait de la déclamation chantante des prêtres ${ }^{20}$ ou des avocats qui se contentaient d'avoir des modulations identiques d'une phrase à l'autre (des tons monotones), les auteurs de la foire et de l'Opéra-Comique tournent à leur tour en dérision, la diction enflée, qui n'est pas en accord avec le sens des vers (le «refrain cyclique», poétique, des tons est en effet détaché de tout contenu).

L'Abbé Joseph Laporte et Jean-Marie-Benoît Clément témoignent des moyens utilisés par certains acteurs pour interpréter les caricatures élaborées par les auteurs. Ainsi Cadoret, jouant sous le nom de Terodac, doit jouer la scène de Métromane dans Acajou. Toute la tirade du personnage est scandée. Outrés, les tragédiens tentent de faire interdire toute parole à la troupe concernée. «L'Auteur de la pièce ne s'en embarrassa guère; \& comme alors les Comédiens déclamaient ou plutôt chantaient, au lieu de réciter naturellement, comme on fait aujourd'hui, l'Auteur nota leur déclamation [...]. La musique de la scène de Métromane s'accordait si bien avec les inflexions et les éclats de voix des Acteurs Tragiques, que l'on s'apercevait à peine de la différence. Ainsi, au lieu de nuire à l'Opéra-Comique, on augmenta son succès par cette singularité $\rangle^{21}$, nous disent Laporte et Clément. Leur propos laisse entendre que la «musique» (la notation) a permis de renforcer l'effet comique de la charge (l'effet est en quelque sorte ultime: les mots sont remplacés par une forme de musique qui reprend la cadence et les «tons» réguliers et monotones des tragédiens). Si les dires des auteurs sont exacts, la déclamation aurait évolué, d'une manière générale, de façon radicale entre 1744 (Acajou) et 1775 (date de la première parution du recueil d'anecdotes). Doit-on finalement penser que les forains ont permis de lancer un débat sur la diction tragique et ont permis à celle-ci d'évoluer dans la mesure où ils furent les premiers à relever les défauts, non pas forcément des tragédiens, mais de la déclamation poétique tragique? Ou ont-ils tout bonnement profité des discussions en cours sur la forme poétique des tragédies, jugée inappropriée par certains dramaturges?

En effet, il semble que les attaques se portent non seulement sur la diction mais aussi sur la forme poétique des pièces. Chaque fois qu'un «déclamateur» (qu'il soit ou non tragédien) paraît en scène, ses paroles sont des vers, quand bien même la pièce est-elle en prose (la déclamation est donc immédiatement reconnaissable). Comme

(19) «Les Ouvrages de l'esprit», (in) Euvres complètes de La Bruyère, éd. J. BENDA, Bibl. de la Pléiade, Paris, Gallimard, 1951, p. 66.

(20) Mazarin soulignait déjà en 1618 que le fait d'adopter des modulations régulièrement identiques ressortissait au théâtre. Il écrivait ainsi dans sa Pratique pour bien prêcher: «Nous mettons au second lieu la coutume de ceux qui font gloire d'observer certains tons en parlant; si bien qu'ils semblent chanter: action qui n'est pas moins importune que celle de crier. [..] Car il faut que la voix soit simple, pure \& résonnante par nature $\&$ non par un industrieux artifice: il faut dis-je éviter un son sauvage \& rustique, sans affecter pourtant un nombre musical, \& harmonieux» (Paris, Jean Méjat, 1618, p. 257).

(21) Abbé J.Laporte et J.-M.-B. Clément, Anecdotes dramatiques, Paris, Duchesne, 1775, p. 8 
le remarque Antoine Houdar de la Motte dans son Discours de la Tragédie dans le premier XVIII ${ }^{e}$ siècle $^{22}$ (le texte date de 1730) lorsqu'il commente l'élaboration de sa tragédie $E$ dipe représentée pour la première fois en 1726, les alexandrins ne permettent pas (et ne sont pas) une manière «naturelle» de parler. Mais il faut de l'extraordinaire sur scène, du pompeux, pour représenter l'univers royal. Les traditions et les conventions scéniques sont tellement ancrées dans la société et chez les acteurs, qu'il ne prend pas le risque de faire représenter sa tragédie en ayant recours à la prose. Il préfère ménager le public habitué à entendre des tragédies versifiées et choisit finalement de conserver l'alexandrin (ce qu'il considère néanmoins comme une certaine forme de lâcheté), ce qui montre - comme d'ailleurs l'affirme encore Luigi Riccoboni en $1738^{23}$, que le public n'est pas forcément contre cette forme de diction au théâtre, voire même qu'il la préfère à une diction moins emphatique qui pourrait résulter d'un phrasé à partir de la prose. Les usages sont acceptés et même toujours prisés dans les années 1720. Par conséquent, choisir d'aller à l'encontre du goût des spectateurs c'est risquer le four et l'humiliation en tant que dramaturge.

Ainsi, si les forains s'avèrent très modernes dans leurs critiques, ils ne sont pas néanmoins nécessairement en accord avec leur temps, en matière de tragique. Ils satisfont une certaine frange du public parisien qui voit dans l'opéra-comique une forme théâtrale originale et inimitable, et qui aime justement ce type de pièce et de représentation pour leur singularité et leur unicité (notamment le mélange musique, chant, comédie, danse). Il n'est guère possible de déterminer si les critiques font mouche à cette époque et si elles ont un écho favorable auprès de l'ensemble des membres du public parisien. Ce sont les théoriciens qui semblent le plus profiter des leçons avant-gardistes de la foire. Il semble en effet que c'est à partir des caricatures des tragédiens (donc d'une diction déformée à l'extrême), qu'est repensé le thêâtre dans sa globalité (type de pièces mais aussi jeu de l'acteur). En effet la seconde raison invoquée par Antoine Houdar de la Motte, en ce qui concerne le maintien de la forme poétique, est la routine des acteurs. Ceux-ci sont eux aussi habitués à interpréter des vers (les tragédies ne sont jamais en prose, sauf rares exceptions comme par exemple au XVII siècle). Ils pourraient se trouver «presque décontenancés dans le tragique s'ils n’y parloient pas en vers» écrit La Motte. Car «leur voix, leur maintien, leur geste, tout est mesuré». Changer l'écriture reviendrait à changer intégralement le système de jeu (chose qui sera contestée plus tard par Marmontel dans son article «Déclamation théâtrale» publié en 1753 dans l'Encyclopédie), et ainsi, les mettre dans l'embarras, la forme noble de l'alexandrin leur permettant de devenir imposants sur scène, et par là même de rendre leur personnage digne et majestueu $x^{24}$. Le vers tragique, de par le style, le registre de langue et la forme, participe du processus d'identification de l'acteur au héros et permet même une meilleure compréhension du texte et par l'acteur, et par le public. Aussi La Motte termine-t-il par ces quelques remarques intéressantes: «D'ailleurs la mesure et les phrases, ordinairement plus coupées dans les vers, aident beaucoup leur intelligence; ils en discernent plus aisément le sens; ils en prennent mieux les tons, et ils les soutiennent davantage; au lieu qu'il leur faudroit plus de finesse que n'en ont quelques-uns, pour saisir dans les phrases étenduës de la prose les inflexions délicates que demanderoient les raisonnemens et les passions $»^{25}$.

(22) A. Houdar de la Motte, Euvres, Paris, Prault, 1754, pp. 390-392. Voir ChaOuCHe, La Philosophie de l'Acteur, édition citée, pp. 201-202.

(23) L. Riccoboni, Pensées sur la déclamation, Paris, Briasson-Delormel-Prault, 1738, p. 35.
(24) Il faut noter que la comédie classique est elle aussi écrite la plupart du temps en vers. D'après Sainte-Albine, le mode de diction est plus souple dans le comique que dans le tragique.

(25) La MotTe, édition citée, p. 392. 
Si les acteurs sont plus aptes à entendre les vers, et sur scène, à les mettre en valeur, doit-on en conclure, dès lors, que la diction tragique respecte mieux le sens du texte (la forme verbale et orale épousant harmonieusement le contenu)? Les forains répondent non. Les témoignages sur Baron et la Lecouvreur laissent entendre que oui. Le célèbre opéra-comique de Charles-Simon Favart, Acajou, auquel nous avons déjà fait allusion, reflète-t-il alors, de manière particulièrement synoptique, les débats entamés depuis les années 1720 puisqu'il remet en cause l'ensemble du système «tragique», condamnant non seulement l'acteur mais aussi le versificateur:

Je vous l'ai déjà di: l'auguste Poësie

Est asservie aux loix de la Géométrie?

Tout versificateur doit sçavoir à propos

Toiser une pensée \& combiner des mots.

Que toujours le bon sens, esclave de la rime,

En forme d'axiome expose un [sic] maxime,

Les vers de tragedie au milieu partagés,

Portant six pieds de long, de niveau sont rangés;

Et pour tout Poëte exact, sur les memes modeles,

Resserre son genie entre deux paralelles.

Je vous ai demontré l'art de construire un vers:

Apprenez maintenant ses usages divers. [...]

Dumoins de déclamer, apprenez la methode,

C'est un talent Seigneur, qui devient à la mode;

Dans cet art méchanique, on aime à s'exercer,

Ecoutez mes leçons, je vais vous y dresser.

Pour faire des Heros une illustre peinture,

N'allez pas fortement imiter la nature,

Sans doute ces Heros n'étoient que des ressorts.

Sachez qu'un Prince Grec, ou qu'un Bourgeois de Rome,

Ne parloit pas jadis de même qu'un autre homme;

Ces Pyrrus, ces Brutus, en perruque en chapeau,

En paniers de baleine \& couverts d'oripeau,

Malgré le sens commun, guidés par la mesure,

Le moindre Confident sur pareil ton monté,

Avoit comme son Maître un langage noté;

Tous parloient en chantant \& leur voix compassée

Ne s'ajustoit qu'au geste, et non à la pensée;

Chaque Acteur pour les peindre \& s'exprimer comme eux,

Dit des vers ampoulés qui tombent deux à deux.

Examinez mon jeu: c'est ainsi que j'avance,

Je prends une attitude $\mathcal{E}$ fort bas je commence,

Ma voix en même temps s'élève par éclats,

Je balance le corps Ej'agite les bras.

Tantôt avec ardeur, je dis à ma Maîtresse:

Pourquoi me fuyez-vous adorable Princesse?

Aux tourmens que j'endure ayez quelques égards;

Cruelle, je mourrai privé de vos regards,

Hélas!... de cet hélas, distinguez l'intervalle.

Tantôt de mes deux bras décrivant un ovale,

J'en impose aux bumains du ton sacré des Rois,

Et je mugis des Vers en étouffant ma voix.

Actrices qui briguez les honneurs de la Scene,

Que dès le premier vers la fureur vous entraîne,

Etendez votre bras pour mieux le faire voir,

Relevez l'estomach, étalez le mouchoir, 
Criez à tout propos, criez à perdre baleine,

Que l'on croye en un mot voir hurler Melpomene .

Par ce goût general, que chacun soit conduit,

On ne doit déclamer que pour faire du bruit.

Taratatantalera... mais quel demon m'inspire!

Quels goufres sont ouverts! Taratantalerire......

Ab Princesse! Ab Seigneur! je deviens furieux...

C'est ainsi qu'en partant je vous fais mes adieux ${ }^{26}$.

La première partie de cet extrait, une fois de plus tiré de la scène 4 de l'acte I, met l'accent sur la rigidité de l'écriture poétique et du genre tragique. L'auteur, dès l'élaboration de la pièce, s'enferme dans un système «mathématique» et «mécanique», qui comporte un certain nombre de divisions régulières, des «combinaisons», mais aussi une homophonie «forcée» (la nécessaire présence de la rime). Le rythme cadencé de l'alexandrin, donne à la déclamation, à la base, un caractère artificiel. On ne s'étonne guère alors de voir un chapitre dans Le Comédien de Pierre Rémond de Sainte-Albine, publié trois ans après la première d'Acajou, intitulé «La Tragédie demande-t-elle à être déclamée?». La seconde partie du texte suggère quant à elle que l'acteur non seulement se plie à la mécanique des vers, mais déclame de manière outrée, commençant bas et terminant sa réplique avec éclats. Ce principe est d'ailleurs décrit dès le XVII ${ }^{e}$ siècle dans les ouvrages consacrés à la rhétorique. Jean Poisson le souligne encore en $1717^{27}$, de même que François Riccoboni en 1750 (soit quelques années après $A(a j o u)^{28}$. «On ne doit déclamer que pour faire du bruit»: ce vacarme sans finesse aucune, cette déclamation extravagante de par ses éclats et sa fureur, sont aussi discutés par Sainte-Albine ${ }^{29}$, puis plus tard par Claude-Joseph Dorat ${ }^{30}$ et par Servandoni D'Hannetaire ${ }^{31}$ (qui commente Sainte-Albine), mais aussi par divers voyageurs européens comme par exemple Thomas Moore ${ }^{32}$. Enfin les descriptions concernant la gestuelle et la disharmonie entre ce qui est dit et ce qui est joué suggèrent que le jeu à la Comédie-Française fonctionne à partir de postures et de gestes conventionnels qui se veulent essentiellement didactiques pour le public, mais qui ne sont pas réellement inspirés par une impulsion ou une illusion intérieure ${ }^{33}$. Or, une

(26) Ch.-S. Favart, Acajou, opéra-comique [imité de «Acajou et Zirphile», de C. Duclos], par M. Favart... [Paris, Théâtre du faux-bourg Saint-Germain, 18 mars 1744.], Paris, Prault fils, 1744, acte I, scène 4 .

(27) J. Poisson, Réflexions sur l'art de parler en public, s.1., 1717, p. 24. «C'est par là qu'une Période dite rapidement, presque d'un même port de voix, \& finie sur un ton un peu emphatique, fait un bel effet au Théâtre, \& que s'attirant un applaudissement général, l'Acteur fait faire ce que nous appelons le Brouhaha».

(28) F. Riccoboni, L'Art du Théâtre à madame de ${ }^{*}$, Paris, C.-F. Simon fils \& Giffart fils, 1750, p. 22. «Commencer bas, prononcer avec une lenteur affectée, traîner les sons en langueur sans les varier, en élever un tout à coup aux demi pauses du sens, \& retourner promptement au ton d'où l'on est parti; dans les moments de passion, s'exprimer avec une force surabondante, sans jamais quitter la même espèce de modulation, voilà comme on déclame».

(29) Voir le chapitre: «Un Comédien peut-il avoir trop de feu?» (Paris, Vincent, Saillant, Desaint, 1747, pp. 41-49).
(30) Cl.-J. Dorat, La Déclamation théâtrale, poème didactique en trois chants, Paris, Sébastien Jorry, 1766, pp. 16-17.

(31) Observations sur l'art du comédien, edition citée, pp. 4-5. «Observez-les, surtout dans un Drame ou dans une Tragédie, vous les verrez, criant à tort et à travers, s'abandonner à une fougue monotone, aussi froide qu'impétueuse, quelquefois même aussi indécente que ridicule: espèce de convulsions que bien de gens prennent pour du feu et des entrailles, mais qui ne sont que le fruit d'une imitation déplacée, maladroite et le plus sottement combinée qu'il soit possible. Ce qu'il y a de singulier, et en même temps de malheureux pour ces Acteurs, c'est qu'ils se trouvent d'autant plus autorisés à ces sortes d'écarts, qu'ils ne sont jamais plus applaudis que dans leur véhémence excessive et dans leurs extinctions de voix».

(32) Voir TH. Moore, Lettres d'un voyageur anglois sur la France, la Suisse et l'Allemagne, Genève, Bardin, 1781 (lettre XVII). Il est curieux de voir dans ces lettres combien le jeu anglais est considéré, lui aussi, comme un «vacarme» faits «d'éclats».

(33) Voir Chaouche, La Philosophie de l'Acteur. édition citée, pp. 213-263 et 295-363. 
fois de plus, les témoignages concernant Mlle Dumesnil à la même période ${ }^{34}$ sur son habileté à faire fi des codes, signalent que certains acteurs de la Comédie-Française s'écartent de la routine et des traditions. C'est aussi précisément à cette époque que les théories anglaises sur l'enthousiasme influencent la France. Jean Monnet qui avait voyagé jusqu'en Angleterre et tenté d'y ouvrir un théâtre, aurait-il décrit des manières de jouer singulières, «modernes», ou tout du moins inhabituelles à ses associés, comme Favart par exemple? Ainsi certaines pièces des forains ont peut-être permis d'accélérer la réflexion sur le lien entre forme et diction, entre genre et interprétation.

On pourrait ajouter pour finir sur ce chapitre, que l'opéra-comique n'est pas exempt d'artificialité dans sa forme en particulier à travers les vaudevilles et par la suite les ariettes. En effet, on utilise aussi des vers mais sans doute perd-on la «mesure» cadencée de ceux-ci grâce à la musique qui atténue l'uniformité de la diction et permet bien évidemment les modulations à travers les différentes notes et airs chantés. Les pièces mettant en scène le tragédien se servent d'airs populaires ou de couplets qui eux aussi jouent purement sur la forme: ainsi de l'air «Tarare, pon, pon» dans La Ressource des Théâtres, qui s'accorde très bien au «tara, tara, tanta» d'Ampulas (Crispin d'ailleurs contrefait Ampulas mélangeant air et diction absurde: «Taratanta, tarare, / Pon, pon») ou l'air «lire, liron, fa, fa» sur lequel chante l'Industrie: «Souvent d'une Tragédie / Je fais une comédie / Ou même un Ballet d'Opéra, lire, lire, lira?/ Liron, fa, fa, fa», puis, parlé: «J'en sais plus d'une propre à cela, la, la / Lire, lire, lira, liron, fa, fa, fa». Dans L'Essai des talents ou les talents comiques, sur l'air «turlutaine» viennent aussi se greffer ces paroles chantées par Arlequin: «Que nous veut cet homme cy / D'un sujet de Melpomene / O turlutaine / L'on n'a pas besoin icy / Turlututu tantalery / N'importe il faut l'écouter». Ajoutons encore les paroles de l'Examinateur, sur l'air «lauderirette»: «Le ton tragique en ce lieu cy / Ne peut causer que de l'ennuy / Landerirette / Temoin monsieur l'Amphigoury / Landeriry» et plus loin, sur l'air «guerlinguinguin»: «N'est-ce point une imposture / Lure lure lure / Cesar se change en Arlequin / Guerlin guin guin». Enfin, les paroles de l'Industrie à la scène 4 de La Ressource des acteurs sont tout autant des syllabes répétées de manière ludique et qui suggèrent que le procédé est exactement le même lorsque l'on joue avec la déclamation tragique: «Il faudra vous appuyer/ De la Pan, pan, pan, / De la to, to, to, / De la mi, mi, mi, / La pan, pan, / La to, to, / La mi, mi, / De la Pantomime». Il en va de même pour les zon, zon, les lon, la lonlaire... récurrents dans les pièces de la foire. «Hohohaha / Et pourquoy donc d'où vient cela / Allés allés ne vous mettez point en peine / Turelure lure et lon lan la / C'est un jeu que tout cela» affirme Thérèse à la scène 3 des Talents comiques. Effectivement on serait porté à croire que toutes ces caricatures ne sont qu'un jeu destiné à plaire au public d'une manière particulièrement efficace, et donc participent d'une forme de marketing visant à élaborer une marque de fabrique, un «label», celui de l'Opéra-comique. Il s'agit peut-être d'un désir secret de s'établir en tant qu'institution (ce qui arrivera par la suite avec Jean Monnet et la création de l'Opéra-Comique), ou tout du moins de s'imposer dans le paysage théâtral parisien, afin de rester «visible» et attirer des spectateurs.

Usant parfois de démagogie en dénaturant les évolutions du jeu tragique dans les années 1720 à 1760, ou faisant acte de propagande à travers une «publicité» mensongère voire une mauvaise presse à l'encontre des tragédiens et des acteurs de la Comédie-Française, les forains ont su initier néanmoins des débats pertinents relatifs au jeu et à l'art de l'acteur (débats qui sont développés tout au long du siècle), comme par exemple la remise en cause de la forme poétique jugée inappropriée pour «imi- 
ter» la nature, de la diction tragique cadencée, ou des emportements et éclats, lors de scènes d'amour et de galanterie, ou lors de simples scènes de délibérations politiques (un prince s'entretenant avec son confident). Un fait demeure cependant inexpliqué. Les débats concernant la forme versifiée, jugée «artificielle», ne semblent pas avoir touché le genre propre de l'Opéra-comique, basé lui aussi en partie sur l'utilisation de la poésie, mais de manière intégralement «musicale» (ou musiquée). Il semble avoir été écarté des théories et ne parait pas avoir servi de modèle dans celles-ci (si ce n'est dans les Lettres à Eugénie de Charles-Joseph de Ligne). On ne fait guère d'allusion au genre unique de l'Opéra-comique, ni même au jeu de ses acteurs, qu'ils soient forains, ou plus tard, comédiens du roi. Doit-on comprendre qu'il s'agissait d'une affaire de snobisme (un «sous-théâtre», ou plutôt un théâtre «non académique», donc indigne d'être étudié et pris en exemple), ou de goût uniquement? La mode étant à un genre nouveau, hybride, n'en voyait-on que l'agrément sans chercher à l'analyser ni même à comparer les types de jeu et l'écriture dramatique des différentes scènes parisiennes? Ainsi ce qui nous semble tout aussi artificiel qu'à la Comédie-Française, passait-il inaperçu, du fait même de sa nouveauté et de sa modernité? En effet, tous ces airs, ces fredons et paroles fondées sur un jeu de son et de syllabes, sur des onomatopées, fonctionnent exactement comme les vers tragiques caricaturés. Ainsi peut-on dès lors vraiment parler de caricature? Ne s'agit-il pas plutôt d'une création, somme toute, originale tant des auteurs que des acteurs? Le génie des forains, nous semble-t-il finalement, est d'avoir élaboré, grâce à l'invention de la parodie de la diction tragique, une forme de vaudeville tant chantée que «parlée-chantée», mais chose encore plus étonnante une forme mélangée de tragique-comique (c'est-à-dire en définitive, à la manière de Rabelais un grotesque joyeux), qui s'accorde parfaitement avec le genre foisonnant de l'opéra-comique mais aussi qui complète parfaitement celui-ci, où manque précisément le tragique/le sérieux. On pourrait ainsi conclure par ces quelques mots de Mme de Staël à propos de la déclamation française: «sur la scène française, on est impatienté quand on n'est pas ému: les sons ampoulés et faux dégoûtent tellement alors de la tragédie, qu'il n'y a pas de parodie, si vulgaire qu'elle soit, qu'on ne préfère à la fade impression du maniérés ${ }^{35}$. A la fin du XVIII ${ }^{\mathrm{e}}$ siècle, ce n'est plus simplement le public populaire qui se veut le défenseur du genre mais aussi les Connaisseurs, signe que les temps changent et qu'une nouvelle époque commence en ce qui concerne l'interprétation proprement dite d'une pièce ${ }^{36}$.

SABINE CHAOUCHE

(35) Madame la baronne Germaine de Staël, De l'Allemagne, chapitre XXVII, (in) Euvres complètes, Paris, Firmin-Didot, p. 141.
(36) Nous tenons à remercier François Moureau pour ses conseils. 